\title{
Quantitative analysis of X-band weather radar attenuation correction accuracy
}

\author{
A. Berne and R. Uijlenhoet \\ Hydrology and Quantitative Water Management, Wageningen University, The Netherlands \\ Received: 20 December 2005 - Revised: 20 February 2006 - Accepted: 25 February 2006 - Published: 2 June 2006
}

\begin{abstract}
At short wavelengths, especially C-, X-, and Kband, weather radar signals are attenuated by the precipitation along their paths. This constitutes a major source of error for radar rainfall estimation, in particular for intense precipitation. A recently developed stochastic simulator of range profiles of raindrop size distributions (DSD) provides a controlled experiment framework to investigate the accuracy and robustness of attenuation correction algorithms. The work presented here focuses on the quantification of the influence of uncertainties concerning radar calibration, the parameterization of power law relations between the integral variables (radar reflectivity $Z$ and specific attenuation $k$ ), and total path integrated attenuation (PIA) estimates at X-band. The analysis concerns single frequency, incoherent and nonpolarimetric radar systems. Two attenuation correction algorithms, based on a forward and a backward implementation respectively, are studied. From DSD range profiles, the corresponding profiles of integral radar variables are derived. Using a Monte Carlo approach, the accuracy and robustness of the two algorithms are quantified for the different sources of error previously mentioned. This framework of realistic DSD variability provides a robust way to confirm that, under realistic assumptions concerning the PIA estimation uncertainty, the forward algorithm outperforms the backward algorithm for PIA values below $10 \mathrm{~dB}$.
\end{abstract}

\section{Introduction}

The space-time variability of rainfall is an important issue in a number of areas, e.g., hydrology, meteorology, and natural hazards. Weather radar is in principle able to provide quantitative estimates of rainfall fields with a high spatial and temporal resolution. Recently, there has been an increased

Correspondence to: R. Uijlenhoet

(remko.uijlenhoet@wur.nl) interest in radars operating at short wavelengths, roughly from 1 to $5 \mathrm{~cm}$. Examples are X-band radar networks (e.g., CASA, http://www.casa.umass.edu) and K-band radars operating from spaceborne platforms (e.g., the TRMM and GPM satellites). Even many operational radar networks across Europe operate at relatively short wavelengths (C-band). At such wavelengths, the attenuation of the radar signal by the precipitation along its path is a critical issue for quantitative radar rainfall estimates that has been recognized for a long time (e.g., Atlas and Banks, 1951). A recently developed stochastic simulator of range profiles of raindrop size distributions (DSD) provides a controlled experiment framework to investigate the accuracy and robustness of attenuation correction algorithms (Berne and Uijlenhoet, 2005).

This paper focuses on the quantification of the influence of uncertainties concerning the radar calibration, the parameterization of a power-law relation between the radar reflectivity $Z$ and the specific attenuation $k$, and total path integrated attenuation (PIA) estimates. The analysis concerns single frequency, incoherent and non-polarimetric radar systems. Two attenuation correction algorithms are studied: a forward algorithm based on the analytical solution proposed by Hitschfeld and Bordan (1954) and a backward algorithm based on the solution proposed by Marzoug and Amayenc (1994). From DSD range profiles, the corresponding profiles of bulk rainfall variables are derived. Using a Monte Carlo approach, the accuracy of the two algorithms is quantified for the different sources of error previously mentioned. The results presented in this paper complement previous work on the sources of uncertainty in attenuation correction (e.g., Delrieu et al., 1999a), by emphasizing on the variability of the DSD along a range profile in a stochastic framework.

Published by Copernicus GmbH on behalf of the European Geosciences Union. 
Table 1. Mean, standard deviation and characteristic spatial scale of $N^{\prime}=\ln N_{t}\left(\right.$ with $N_{t}$ in $\mathrm{m}^{-3}$ ) and $\lambda^{\prime}=\ln \lambda$ (with $\lambda$ in $\left.\mathrm{mm}^{-1}\right)$ deduced from HIRE' 98 data at a $2 \mathrm{~s}$ time step.

\begin{tabular}{cccc}
\hline & Mean & Std & $\theta(\mathrm{km})$ \\
\hline$N^{\prime}$ & 8.11 & 0.41 & 4.4 \\
$\lambda^{\prime}$ & 0.93 & 0.31 & 4.4 \\
\hline
\end{tabular}

\section{DSD simulator}

The DSD simulator used in the following has been proposed by Berne and Uijlenhoet (2005). It enables to generate realistic DSD range profiles. It is based on the exponential DSD, which two parameters $N_{t}$ and $\lambda$ are considered to be random variables

$N\left(D \mid N_{t}, \lambda\right)=N_{t} \lambda e^{-\lambda D}$,

where $N\left(D \mid N_{t}, \lambda\right) d D$ denotes the drop concentration in the diameter interval $\left[D, D+d D\right.$ ] given $N_{t}$ (total drop concentration) and $\lambda$. The latter are assumed to be jointly lognormally distributed. In order to be able to simulate spatial correlation within the range profiles, $N^{\prime}=\ln N_{t}$ and $\lambda^{\prime}=\ln \lambda$ are assumed to follow a first order discrete vector auto-regressive process:

$\mathbf{X}[j+1]=\mathbf{C}_{\mathbf{1}} \mathbf{C}_{\mathbf{0}}{ }^{-1} \mathbf{X}[j]+\mathbf{E}[j+1]$,

where

$$
\begin{aligned}
\mathbf{X}[j] & =\left[\begin{array}{c}
N^{\prime}(j)-\mu_{N^{\prime}} \\
\lambda^{\prime}(j)-\mu_{\lambda^{\prime}}
\end{array}\right], \\
\mathbf{C}_{\mathbf{0}} & =\left[\begin{array}{cc}
\sigma_{N^{\prime}}^{2} & \sigma_{N^{\prime}} \sigma_{\lambda^{\prime}} \rho_{N^{\prime} \lambda^{\prime}} \\
\sigma_{N^{\prime}} \sigma_{\lambda^{\prime}} \rho_{N^{\prime} \lambda^{\prime}} & \sigma_{\lambda^{\prime}}^{2}
\end{array}\right], \\
\mathbf{C}_{\mathbf{1}} & =\left[\begin{array}{cc}
\sigma_{N^{\prime}}^{2} \rho_{N^{\prime}}(1) & \sigma_{N^{\prime}} \sigma_{\lambda^{\prime}} \rho_{N^{\prime} \lambda^{\prime}}(1) \\
\sigma_{N^{\prime}} \sigma_{\lambda^{\prime}} \rho_{\lambda^{\prime} N^{\prime}}(1) & \sigma_{\lambda^{\prime}}^{2} \rho_{\lambda^{\prime}}(1)
\end{array}\right], \\
\mathbf{E}[j+1] & =\left[\begin{array}{c}
\epsilon_{N^{\prime}}(j+1) \\
\epsilon_{\lambda^{\prime}}(j+1)
\end{array}\right],
\end{aligned}
$$

$j$ is the distance index, $\rho_{N^{\prime}}(1)$ represents the autocorrelation at ag 1 (idem for $\left.\lambda^{\prime}\right), \rho_{N^{\prime} \lambda^{\prime}}(1)$ represents the cross-correlation at lag 1 , and $\epsilon_{N^{\prime}}$ represents a Gaussian white noise process (idem for $\lambda^{\prime}$ ). Therefore $\mathbf{C}_{\mathbf{0}}$ and $\mathbf{C}_{\mathbf{1}}$ are the covariance matrices at lags 0 and 1 . The variances of the white noise processes $\epsilon_{N^{\prime}}$ and $\epsilon_{\lambda^{\prime}}$ are fixed such that $\mathbf{X}$ is a second order stationary process. For such a first order vector auto-regressive process, the auto-correlation function takes an exponential form according to:

$\rho(r)=e^{-2 r / \theta}$, where $r$ represents the distance lag and $\theta$ the characteristic spatial scale, also known as the scale of fluctuation (Vanmarcke, 1983):

$\theta=2 \int_{0}^{\infty} \rho(r) d r$.

Using this stochastic model, we are able to generate range profiles of DSDs of equivolumetric spherical drops. DSD time series measurements from an optical spectropluviometer, collected during the HIRE'98 experiment (Uijlenhoet et al., 1999) in Marseille, France, are used to parameterize the model. We focus on a period of $45 \mathrm{~min}$ of intense rainfall during the 7 September 1998 rain event in order to simulate high rainfall intensities. To convert the measured DSD time series to DSD range profiles, we assume Taylor's hypothesis with a constant velocity of $12.5 \mathrm{~m} \mathrm{~s}^{-1}$, consistent with the wind speed estimate of Berne et al. (2004). The zerolag cross-correlation between the fitted $N^{\prime}$ and $\lambda^{\prime}$ values is found to be negligible. The characteristic scale $\theta$ is found to be very similar for $N^{\prime}$ and $\lambda^{\prime}$, and is therefore assumed to be equal. Note that this is not a prerequisite of the model. As a consequence, the number of model parameters reduces to five: the mean and standard deviation of $N^{\prime}$ and $\lambda^{\prime}$, and the characteristic scale $\theta$. Their values are given in Table 1 .

The generated DSD profiles have a total length of $30 \mathrm{~km}$, with a spatial resolution of $25 \mathrm{~m}$ (corresponding to a $2 \mathrm{~s}$ time step). From these DSD profiles, the corresponding profiles of bulk rain variables (radar reflectivity $Z$ and specific one-way attenuation $k$ ) are easily derived, using the Mie theory (van de Hulst, 1981) for the scattering cross-sections and Beard's velocity model for the drop terminal fall velocities (Beard, 1976). The radar reflectivity $Z\left[\mathrm{~mm}^{6} \mathrm{~m}^{-3}\right]$ is defined as

$Z=\frac{10^{6} l^{4}}{\pi^{5}|K|^{2}} \int_{0}^{\infty} \sigma_{B}(D) N\left(D \mid N_{t}, \lambda\right) d D$

where $l[\mathrm{~cm}]$ denotes the wavelength of the radar signal, $K$ [] is a constant related to the refractive index of the hydrometeors, and $\sigma_{B}\left[\mathrm{~cm}^{2}\right]$ is the backscattering cross-section. Similarly, the specific one-way attenuation $k\left[\mathrm{~dB} \mathrm{~km}^{-1}\right]$ is defined as

$k=\frac{1}{\ln 10} \int_{0}^{\infty} \sigma_{E}(D) N\left(D \mid N_{t}, \lambda\right) d D$

where $\sigma_{E}\left[\mathrm{~cm}^{2}\right]$ is the extinction cross-section. Figure 1 presents an example of generated $N_{t}$ and $\lambda$ profiles, as well as the corresponding attenuated and non-attenuated $Z$ profiles at X-band. This controlled experiment framework allows to apply a Monte Carlo technique to quantify the respective influence of the different sources of uncertainty in attenuation correction. 


\section{Attenuation correction algorithms}

As mentioned in the introduction, we consider incoherent, single frequency and non-polarimetric radar systems. Two different types of algorithms will be studied in the following. The measured attenuated reflectivity $Z_{a}$ reads

$Z_{a}(r)=\delta_{c} A(r) Z(r)$,

where $\delta_{c}$ is the calibration error factor and $A(r)$ is the twoway attenuation factor at the range $r(0 \leq A \leq 1)$. Assuming the $Z-k$ relation reads

$Z=\delta_{\alpha} \alpha k^{\delta_{\beta} \beta}$,

where $\delta_{\alpha}\left(\delta_{\beta}\right.$ respectively) is the error factor in $\alpha(\beta)$. Therefore, $A$ can be written as

$A(r)=\exp \left[-0.2 \ln (10) \int_{0}^{r}\left(\frac{Z(s)}{\delta_{\alpha} \alpha}\right)^{1 /\left(\delta_{\beta} \beta\right)} d s\right]$.

Hitschfeld and Bordan (1954) (HB hereafter) proposed an analytical solution to express $Z$ as a function of $Z_{a}$ :

$$
\begin{aligned}
& Z(r)=Z_{a}(r) / \\
& {\left[\delta_{c}^{1 /\left(\delta_{\beta} \beta\right)}-\frac{0.2 \ln (10)}{\delta_{\beta} \beta} \int_{0}^{r}\left(\frac{Z_{a}(s)}{\delta_{\alpha} \alpha}\right)^{1 /\left(\delta_{\beta} \beta\right)} d s\right]^{\delta_{\beta} \beta} .}
\end{aligned}
$$

The HB algorithm is a forward algorithm because the integral is between 0 and $r$. However, the difference in its denominator can be close to 0 and this makes the algorithm potentially highly unstable (Hitschfeld and Bordan, 1954).

To avoid instability problems, another family of attenuation correction algorithms has been developed. It is based on the knowledge of an estimate $A_{0}$ of the PIA at a given range $r_{0}$. For ground based radar, ground echoes may be used to derive PIA estimates by comparing their reflectivity values during dry and rainy periods, as proposed by Delrieu et al. (1997). The estimate $A_{0}$ can be uncertain, that is

$A\left(r_{0}\right)=\delta_{A} A_{0}$,

where $\delta_{A}$ is the error factor in $A_{0}$. The reformulation of Eq.(10) starting from $r_{0}$ and going backward to the radar guarantees the stability of the algorithm. As an example, we use the solution proposed by Marzoug and Amayenc (1994) (MA hereafter):

$$
\begin{aligned}
Z(r)= & Z_{a}(r) / \\
& {\left[\left(\delta_{c} \delta_{A} A_{0}\right)^{1 /\left(\delta_{\beta} \beta\right)}\right.} \\
& \left.+\frac{0.2 \ln (10)}{\delta_{\beta} \beta} \int_{r}^{r_{0}}\left(\frac{Z_{a}(s)}{\delta_{\alpha} \alpha}\right)^{1 /\left(\delta_{\beta} \beta\right)} d s\right]^{\delta_{\beta} \beta} .
\end{aligned}
$$

The main drawback of such a backward algorithm is that it requires a reliable estimation of the PIA at a given range.

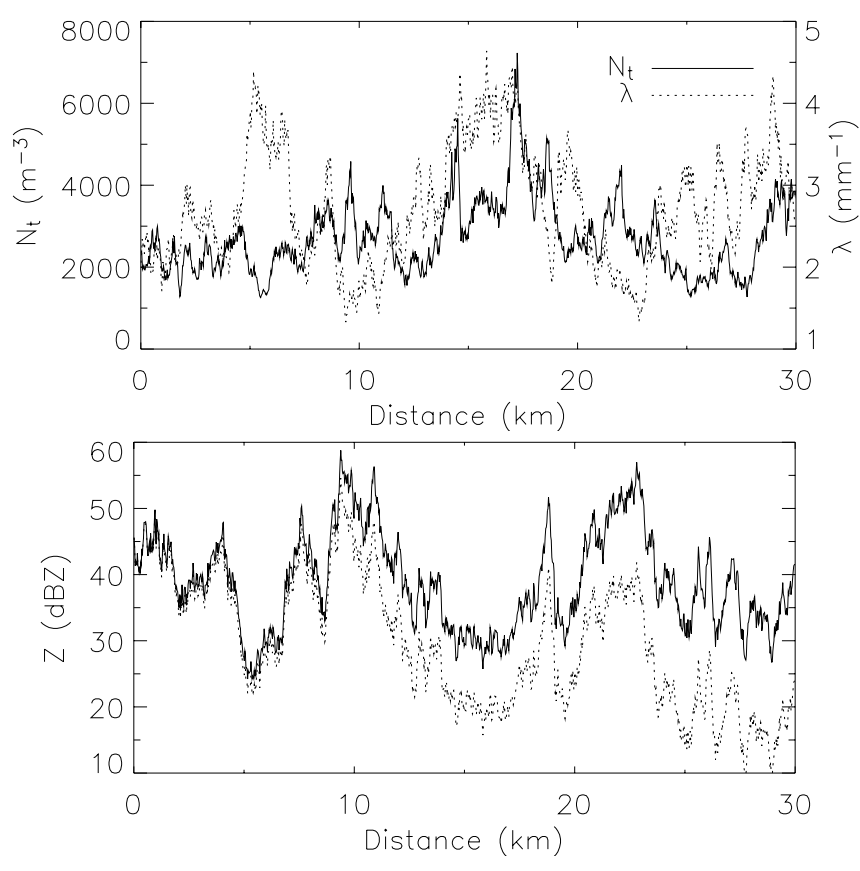

Fig. 1. Top panel: example of generated $N_{t}$ and $\lambda$ profiles. Bottom panel: corresponding non-attenuated $Z$ (solid) and attenuated $Z_{a}$ (dashed) profiles at X-band frequency.

\section{Monte Carlo approach}

To study the accuracy of the algorithms, we use a Monte Carlo technique. The analysis focuses on attenuation correction at X-band (3.2 cm wavelength) using Eqs. (10) and (12). One thousand profiles of $N_{t}$ and $\lambda$ (hence of $Z, k$ and $Z_{a}$ ) are generated. To be consistent with operational radar sampling resolutions, the high spatial resolution $(25 \mathrm{~m})$ profiles are averaged at a lower spatial resolution of $250 \mathrm{~m}$. On each profile a $Z-k$ power-law relation is fitted by means of a non-linear regression technique. It must be noted that they constitute the best possible power-law relations. Figure 2 presents the distributions of prefactor and exponent values for the 1000 profiles. The exact PIA value is calculated as the difference between the non-attenuated and the attenuated $\mathrm{Z}$ profiles. Then the two algorithms are applied using the fitted relations on the 1000 profiles. Using these reference values enables to independently analyze the influence of the different sources of error on the two attenuation correction algorithms.

The top pannel of Fig. 3 shows the median, as well as the $10 \%$ and $90 \%$ quantiles, of the distribution of the root mean square error (RMSE) calculated between the exact $Z$ profiles and the $Z_{c}$ profiles obtained by applying the two attenuation correction algorithms without any uncertainty (i.e. $\delta_{c}=\delta_{\alpha}=\delta_{\beta}=\delta_{A}=1$ ). To illustrate the effect of attenuation, the RMSE between the $Z$ and the uncorrected $Z_{a}$ profiles has also been plotted, and appears to be much larger than the 

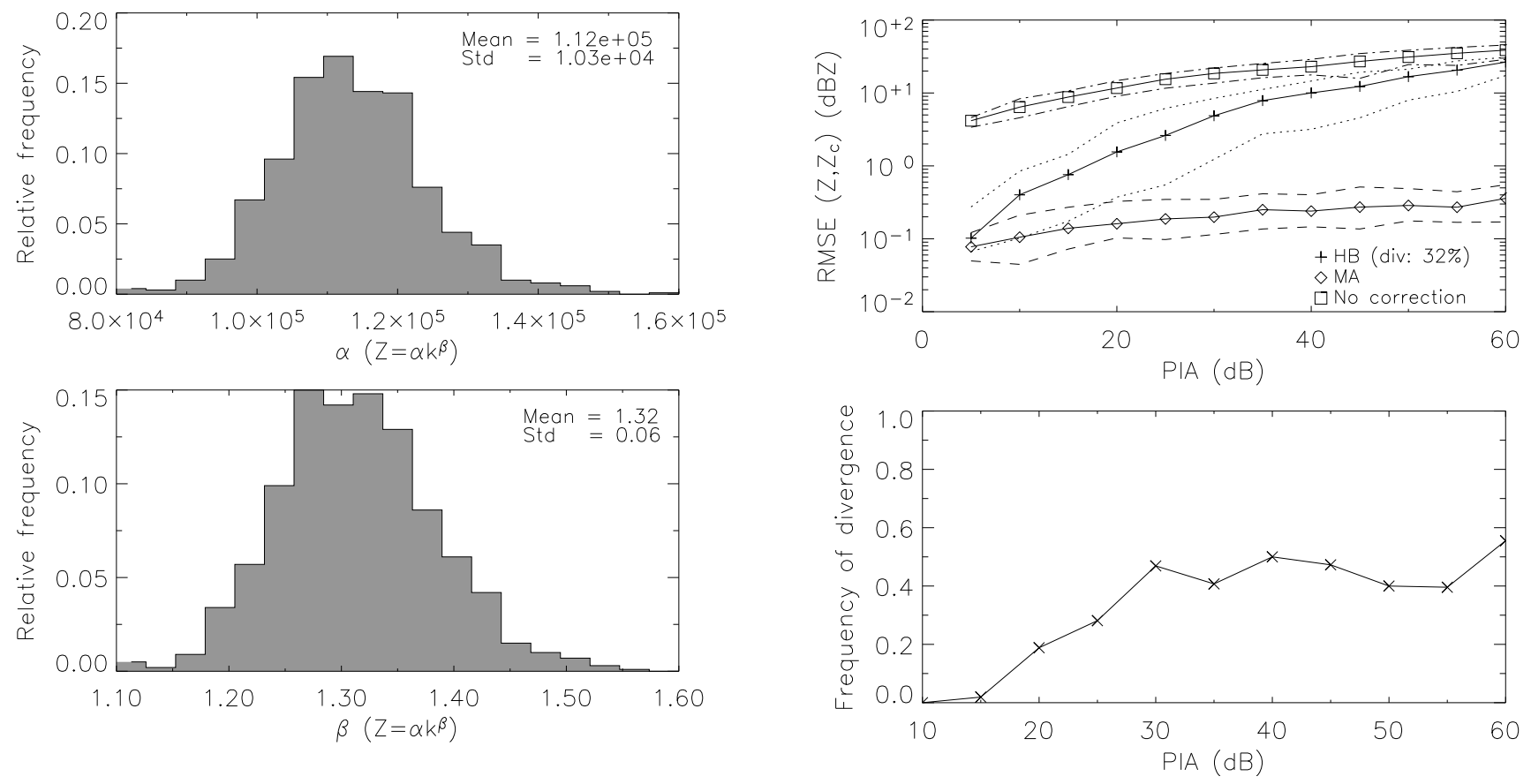

Fig. 2. Distribution of the values of the prefactor $\alpha$ (top panel) and exponent $\beta$ (bottom panel) of the $Z$ - $k$ relation for the 1000 profiles at X-band frequency.

RMSE for the $Z_{c}$ profiles. The significant dispersion of the distribution (top panel of Fig. 3 is in log scale) is explained by the fact that the use of a deterministic power law between $Z$ and $k$ is not consistent with the stochastic nature of these variables.

As a well-known result (e.g., Marzoug and Amayenc, 1994), the MA algorithm $(0.1<$ median $<0.3 \mathrm{dBZ})$ is more stable and accurate than the HB algorithm $(0.1<$ median $<20 \mathrm{dBZ})$, which additionally diverges in about 1 in 3 cases in total. The bottom panel of Fig. 3 shows the percentage of divergent cases as a function of the PIA value. This confirms previous work on the instability of the HB algorithm (e.g, Delrieu et al., 1999a), showing that the $\mathrm{HB}$ algorithm becomes significantly unstable when the PIA is above $15 \mathrm{~dB}: 20 \%$ (40\%) of divergence for a PIA of about $20 \mathrm{~dB}(30 \mathrm{~dB})$. The RMSE values in the top panel of Fig. 3 will constitute the reference values for the quantification of the influence of the different sources of uncertainty, as detailed in the following sections.

\section{Influence of the uncertainty in calibration}

Radar systems can be affected by calibration errors. In this section, the influence of the uncertainty in calibration on the accuracy of the attenuation correction algorithms is quantified. For better visual inspection, the calibration error is

Fig. 3. Top panel: median (solid line) of the distribution of the RMSE calculated between the exact $Z$ profiles and the attenuated reflectivity $Z_{a}$ ("No correction"), and the $Z_{c}$ profiles obtained by applying the two attenuation correction algorithms ("HB" and "MA"), for 1000 profiles at a $250 \mathrm{~m}$ resolution. The dotted and/or dashed lines represent the $10 \%$ and $90 \%$ quantiles. "div" indicates the percentage of diverging HB corrections. We arbitrarily limit the maximum PIA at $60 \mathrm{~dB}$ for clarity, although $10 \%$ of the profiles have a PIA above $60 \mathrm{~dB}$. The percentage of divergent cases corresponds to all 1000 profiles. The convention is the same for the subsequent figures. Bottom panel: percentage of profiles for which the HB algorithm diverges as a function of the PIA value.

expressed in $\mathrm{dB}$ as $\epsilon_{c}=10 \log \left(\delta_{c}\right)$ and varies in the interval $[-5,+5]$. The additional error due to uncertain calibration is calculated as the ratio between the RMSE values for a given calibration error and the reference RMSE values. Figure 4 presents the median, as well as the $10 \%$ and $90 \%$ quantiles, of the distribution of the RMSE ratio as a function of the calibration error. The other error factors $\left(\delta_{\alpha}, \delta_{\beta}\right.$ and $\left.\delta_{A}\right)$ are fixed to 1 .

As expected, the RMSE ratio rapidly increases when $\epsilon_{c} \neq 0$, that is the calibration error significantly decreases the accuracy of the two algorithms. For instance, the median RMSE ratio value is about 2 when $\epsilon_{c}= \pm 1$ for the MA algorithm. It is about 3 when $\epsilon_{c}=+1$ for the HB algorithm. When $\epsilon_{c}>0$, the median values are similar for the two algorithms but the dispersion is larger for the HB algorithm. When $\epsilon_{c}<0$, the distribution remains similar for the MA algorithm. For the HB algorithm, Eq. (10) shows that $\delta_{c}<1$ (or $\epsilon_{c}<0$ ) results in more diverging profiles because the denominator can 


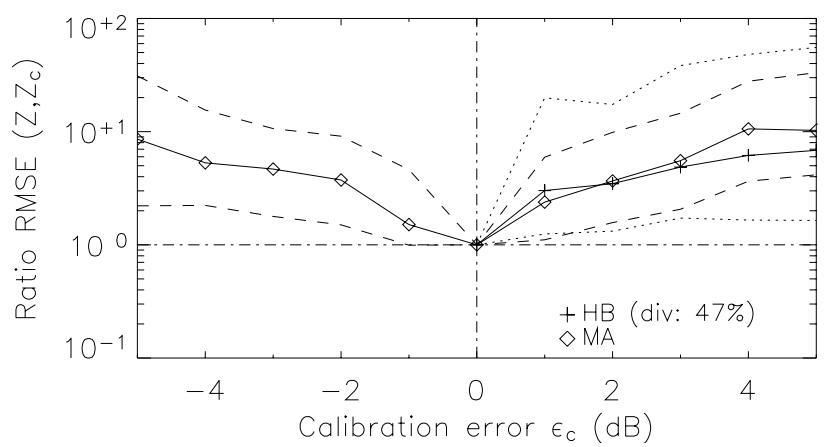

Fig. 4. Median, $10 \%$ and $90 \%$ quantiles of the distribution of the RMSE ratio as a function of the calibration error $\epsilon_{c}$ expressed in $\mathrm{dB}$, for the two attenuation correction algorithms.

approach zero already for smaller $Z_{a}$ values, as illustrated in Fig. 4 by the almost systematic divergence and therefore near complete failure of the $\mathrm{HB}$ algorithm when $\epsilon_{c}<0$ (absence of points and larger percentage of divergences).

\section{Influence of the uncertainty in the parameterization of the $Z-k$ relation}

The two studied algorithms are based on the assumption of a power-law relation between $Z$ and $k$. To analyze the influence of the uncertainty in the parameters of the $Z-k$ power law on the accuracy of the two attenuation correction algorithms, an error factor between 0.7 and 1.3 is applied to the prefactor (the exponent respectively). The additional error due to uncertain parameterization of the $Z-k$ relation is calculated as the ratio between the RMSE values for a given prefactor (exponent) error and the reference RMSE values. Figure 5 presents the median, as well as the $10 \%$ and $90 \%$ quantiles, of the distribution of the RMSE ratio as a function of the relative deviation of the prefactor and exponent, with respect to the reference $Z-k$ relation. The other error factors $\left(\delta_{c}\right.$ and $\left.\delta_{A}\right)$ are fixed to 1 .

For the prefactor (top panel of Fig. 5), the distribution of the RMSE ratio is roughly similar for the two algorithms. The distribution of the RMSE ratio for $\delta_{\alpha}<1$ remains similar for the MA algorithm, while the HB algorithm is more sensitive to an underestimation of the prefactor $\left(\delta_{\alpha}<1\right)$, with a larger dispersion of the quantiles. When the underestimation of $\delta_{\alpha}$ becomes large, the HB algorithm diverges (see Eq.10). The median RMSE ratio is about 3 when the error is about $\pm 15 \%$ in the prefactor for the MA algorithm. It is also about 3 when the error is about $+15 \%$ for the HB algorithm.

The influence of the exponent appears to be similar to that of the prefactor for the HB algorithm, while it is stronger for the MA algorithm. For instance, the median RMSE ratio is about 10 when the error in the exponent is about $\pm 15 \%$ for
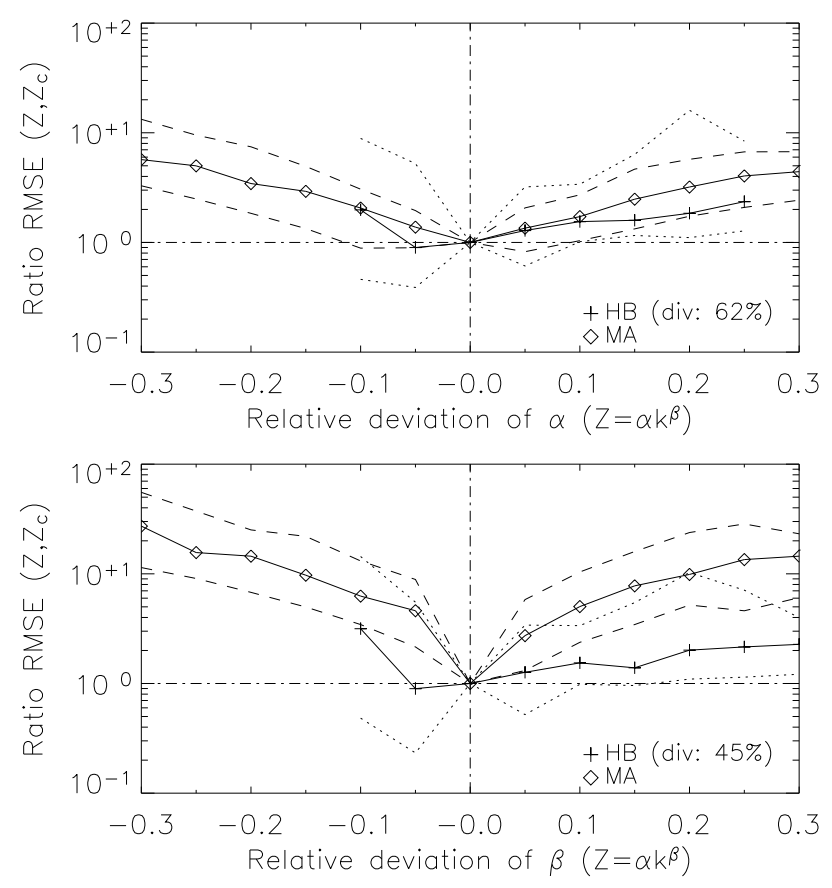

Fig. 5. Median, $10 \%$ and $90 \%$ quantiles of the distribution of the RMSE ratio as a function of the relative deviation of the prefactor (top panel) and exponent (bottom panel) of the $Z-k$ power law, for the two attenuation correction algorithms.

the MA algorithm. It is about 2 when the error is about $15 \%$ for the HB algorithm.

\section{Influence of the uncertainty in the PIA estimate}

The MA algorithm is more accurate and more robust than the $\mathrm{HB}$ algorithm, but it requires an additional parameter which is the estimate of the PIA at a given range. This section is devoted to the quantification of the influence of the uncertainty in this PIA estimate on the accuracy of the MA algorithm. Similarly to $\epsilon_{c}$, we define $\epsilon_{A}=10 \log \left(\delta_{A}\right)$. The error in the PIA estimate $\epsilon_{A}$ is generated as a Gaussian white noise with a standard deviation of $2.5 \mathrm{~dB}$ (Delrieu et al., 1999b). The additional error due to an uncertain PIA estimate $\epsilon_{A}$ is calculated as the ratio between the RMSE values for the uncertain PIA estimate and the reference RMSE values. The top panel of Fig. 6 presents the median, as well as the $10 \%$ and $90 \%$ quantiles, of the distribution of the RSME ratio as a function of $\epsilon_{A}$ for the MA algorithm. The other error factors $\left(\delta_{c}, \delta_{\alpha}\right.$ and $\delta_{\beta}$ ) are fixed to 1 .

According to Eq. (12), the top panels of Fig. 6 and Fig. 4 should in theory be identical as far as the MA algorithm is concerned, because $\delta_{A}$ and $\delta_{c}$ can be interchanged. In practice, for a given reference $Z_{a}$ profile, the error in the calibration and in the PIA estimate are generally different 

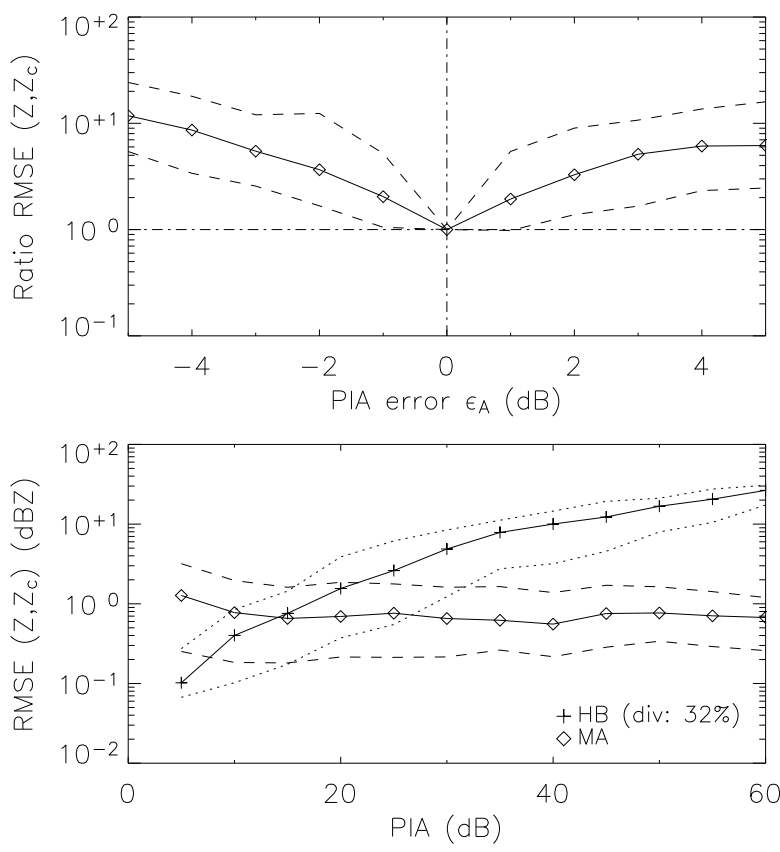

Fig. 6. Top panel: median, $10 \%$ and $90 \%$ quantiles of the distribution of the RMSE ratio as a function of the PIA error $\epsilon_{A}$ expressed in $\mathrm{dB}$ for the MA algorithm. Bottom panel: similar to top panel of Fig. 3, to compare HB and MA with uncertain PIA estimates.

and therefore the deduced distribution of the RMSE ratio is slightly different. Nevertheless, the influence of $\delta_{A}$ is similar to that of $\delta_{c}$.

Finally, it must be noted that an under- or over-estimation of about $2 \mathrm{~dB}$ in the estimated PIA leads to a multiplication of the error by a factor of about 4 . In case of relatively small PIA values (below $10 \mathrm{~dB}$ ), the bottom panel of Fig. 6 shows that the MA algorithm is less accurate than the HB algorithm. This behaviour is consistent with previous work (e.g., Marzoug and Amayenc, 1994; Delrieu et al., 1997). This also suggests that at $\mathrm{X}$-band, one should use the $\mathrm{HB}$ algorithm for small PIA (below $10 \mathrm{~dB}$ ) and the MA algorithm for larger PIA (above $10 \mathrm{~dB}$ ).

\section{Conclusions}

Attenuation correction is an important step for quantitative rain estimation using $\mathrm{C}-$, $\mathrm{X}$-, or $\mathrm{K}$-band weather radar. In this paper, we focus on X-band incoherent, single frequency and non-polarimetric radar systems. We investigate the influence of uncertainties in the radar calibration, in the parameterization of a power-law relation between the radar reflectivity $Z$ and the specific attenuation $k$, and in the total path integrated attenuation (PIA) estimates on the accuracy of two attenuation correction algorithms. The first (HB algorithm) is based on a forward implementation and is known for its instability.
The second (MA algorithm) is based on a backward implementation and is stable, but requires an additional piece of information, which is the PIA at a given range from the radar. A stochastic model of DSD range profiles provides a controlled experiment framework, with fully consistent $Z$ and $k$ profiles, to quantify the influence of the different sources of uncertainty. An uncertainty of $1 \mathrm{dBZ}$ in the measured $Z$ (or of $1 \mathrm{~dB}$ in the PIA estimate) leads to a multiplication of the RMSE by at least a factor 2 with respect to attenuation correction errors resulting from the stochastic nature of the DSD alone. An uncertainty of about $15 \%$ in the prefactor of the $Z-k$ relation leads to a multiplication of the RMSE by at least a factor 3 for both algorithms. The MA algorithm is more sensitive to uncertainties in the exponent (a multiplication by a factor of about 10 for $15 \%$ uncertainty), while the HB algorithm diverges more often.

For practical applications, a combination of the two algorithms appears as a good compromise: in case of small PIA (below $10 \mathrm{~dB}$ ), the HB algorithm should be used, while the MA algorithm should be used when the PIA is larger than $10 \mathrm{~dB}$. Such an hybrid algorithm has been implemented in the context of intense Mediterranean precipitation and has provided reliable results (Delrieu et al., 1997; Berne et al., 2005).

Finally, the framework described in this paper offers a suitable test-bed for other hybrid (e.g., Iguchi and Meneghini, 1994) or polarimetric (e.g., Testud et al., 2000) attenuation correction algorithms.

Acknowledgements. The authors thank G. Delrieu and an anonymous reviewer for their helpful comments. This research is supported by the EU projects FLOODSITE (GOCE-CT-2004505420) and VOLTAIRE (EVK2-2001-00273). The second author is also supported by the Netherlands Organization for Scientific Research (NWO, grant 016.021.003, Vernieuwingsimpuls). This paper reflects the authors' views and not those of the European Community. Neither the European Community nor any member of the FLOODsite Consortium is liable for any use of the information in this paper.

Edited by: P. P. Alberoni

Reviewed by: G. Delrieu and another referee

\section{References}

Atlas, D. and Banks, H.: The interpretation of microwave reflections from rainfall, J. Meteorol., 8, 271-282, 1951.

Beard, K.: Terminal velocity and shape of cloud and precipitation drops aloft, J. Atmos. Sci., 33, 851-864, 1976.

Berne, A. and Uijlenhoet, R.: A stochastic model of range profiles of raindrop size distributions: application to radar attenuation correction, Geophys. Res. Lett., 32, L10803, doi:10.1029/2004GL021899, 2005.

Berne, A., Delrieu, G., Creutin, J.-D., and Obled, C.: Temporal and spatial resolution of rainfall measurements required for urban hydrology, J. Hydrol., 299, 166-179, 2004. 
Berne, A., Delrieu, G., and Andrieu, H.: Estimating the vertical structure of intense Mediterranean precipitation using two Xband weather radar systems, J. Atmos. Oceanic Technol., 22, 1656-1675, 2005.

Delrieu, G., Caoudal, S., and Creutin, J.-D.: Feasibility of using mountain return for the correction of ground-based X-band weather radar data, J. Atmos. Oceanic Technol., 14, 368-385, 1997.

Delrieu, G., Hucke, L., and Creutin, J.: Attenuation in rain for Xand C-band weather radar systems: sensitivity with respect to the drop size distribution, J. Appl. Meteorol., 38, 57-68, 1999a.

Delrieu, G., Serrar, S., Guardo, E., and Creutin, J.-D.: Rain measurement in hilly terrain with X-band weather radar systems: accuracy of path-integrated attenuation estimates derived from mountain returns, J. Atmos. Oceanic Technol., 16, 405-415, $1999 \mathrm{~b}$.

Hitschfeld, W. and Bordan, J.: Errors inherent in the radar measurement of rainfall at attenuating wavelengths, J. Meteorol., 11, 58-67, 1954.

Iguchi, T. and Meneghini, R.: Intercomparison of single-frequency methods for retrieving a vertical rain profile from airborne or spaceborne radar data, J. Atmos. Oceanic Technol., 11, 15071516, 1994.
Marzoug, M. and Amayenc, P.: A class of single- and dualfrequency algorithms for rain-rate profiling from a spaceborne radar, Part I: principle and tests from numerical simulations, J. Atmos. Oceanic Technol., 11, 1480-1506, 1994.

Testud, J., Le Bouar, E., Obligis, E., and Ali-Mehenni, M.: The rain profiling algorithm applied to polarimetric weather radar, J. Atmos. Oceanic Technol., 17, 332-356, 2000.

Uijlenhoet, R., Andrieu, H., Austin, G., Baltas, E., Borga, M., Brilly, M., Cluckie, I., Creutin, J.-D., Delrieu, G., Deshons, P., Fatorelli, S., Griffith, R., Guarnieri, P., Han, D., Mimikou, M., Monai, M., Porrà, J., Sempere-Torres, D., and Spagni, D.: HYDROMET Integrated Radar Experiment (HIRE): experimental setup and first results, in: 29th Int. Conf. On Radar Meteorology, AMS, 926-930, Montral, Canada, 1999.

van de Hulst, H.: Light scattering by small particles, Dover, 1981.

Vanmarcke, E.: Random fields: analysis and synthesis, MIT Press, 1983. 